
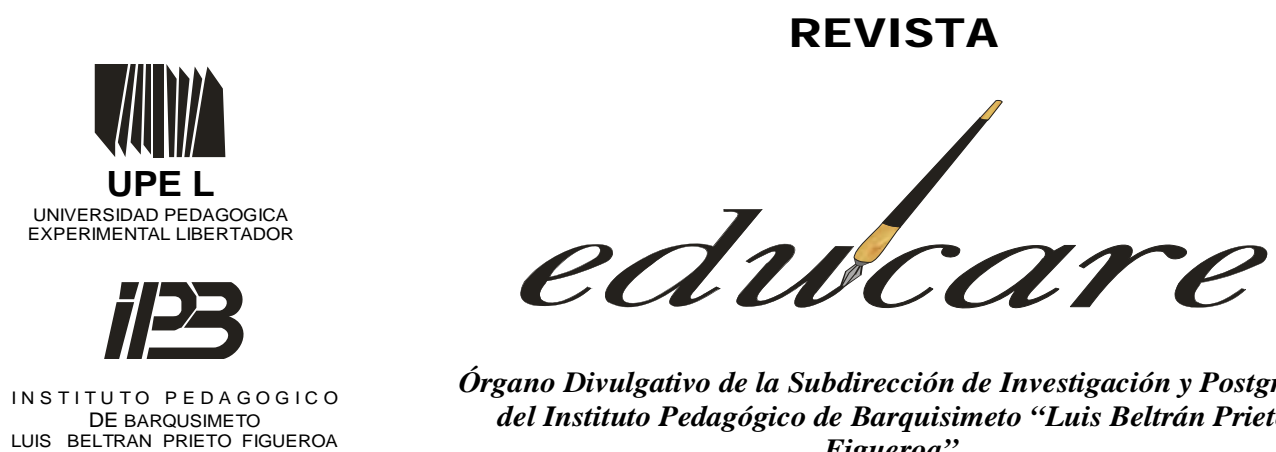

Órgano Divulgativo de la Subdirección de Investigación y Postgrado del Instituto Pedagógico de Barquisimeto "Luis Beltrán Prieto LUIS BELTRAN PRIETO FIGUEROA Figueroa”

BARQUISIMETO - EDO. LARA - VENEZUELA

NUEVA ETAPA

FORMATO ELECTRÓNICO

DEPOSITO LEGAL: ppi201002LA3674

Volumen $18 \mathrm{~N}^{\circ} 1$

ISSN: 2244-7296

Enero - Abril 2014

\title{
EL DEBATE COOPERATIVO VERSUS LA EXPOSICIÓN VERBAL DEL DOCENTE
}

\section{THE COOPERATIVE DEBATE VERSUS TEACHER'S VERBAL EXPOSITION}

\author{
Gilberto Aranguren Peraza*
}

\begin{abstract}
*UNIVERSIDAD PEDAGÓGICA EXPRIMENTAL LIBERTADOR - INSTITUTO PEDAGÓGICO DE CARACAS (UPEL - IPC) UNIVERSIDAD NACIONAL EXPERIMENTAL SIMÒN RODRÍGUEZ (UNESR)
\end{abstract}




\section{EL DEBATE COOPERATIVO VERSUS LA EXPOSICIÓN VERBAL DEL DOCENTE}

THE COOPERATIVE DEBATE VERSUS TEACHER'S VERBAL EXPOSITION

\section{TRABAJO DE INVESTIGACIÒN}

Recibido:27-09-13

\author{
Gilberto Aranguren Peraza* \\ UPEL-IPC UNERS
}

Aceptado:11-02-14

\section{RESUMEN}

La investigación tiene como objetivo el comparar el alcance de dos estrategias de intervención en el aula: el debate cooperativo y la exposición verbal del docente. Se hizo uso de una investigación de campo y del método cuantitativo mediante un diseño de dos grupos (experimental y control) con sujetos no aleatorizados y con post - pruebas. Las estrategias fueron aplicadas en un 9no grado de Educación Básica de Adultos en el área de Ciencias Biológicas. Los resultados son lo suficientemente significativos para señalar que sí existe relación entre las calificaciones obtenidas por los estudiantes y su participación en las estrategias, en un nivel de confianza de $\alpha$ $=0,05$; las discusiones e interacción dadas en los equipos de debate cooperativos ayudan a mejorar el rendimiento, a diferencia de las exposiciones reiteradas del docente. Una conclusión es que las interacciones contribuyen a generar conflictos socio - cognitivos.

Descriptores: El debate cooperativo. La exposición verbal. Los conflictos socio cognitivos.

\begin{abstract}
The present research aims to compare the reach of two intervention strategies in the classroom: cooperative debate and teacher's verbal exposition. It was a quantitative field research with a two groups (control and experimental), pretests and posttest with non-randomized subjects design. The strategies were used in a Biology 9th grade adult's classroom. Results are significant enough to highlight that there exists relationship between students' grades and their participation in the strategies at a confidence interval of $\alpha=0,05$. Discussions and interactions occurring in the cooperative debate groups help to improve students' performance better than continuous teacher's expositions. A conclusion is that interactions contribute to generate socio-cognitive conflicts.
\end{abstract}

Keywords: cooperative debate, verbal exposition, socio-cognitive conflicts.

\footnotetext{
* Doctor en Ciencias de la Educación y Magister en Ciencias de la Educación. Profesor del Área de Postgrado del Núcleo de Los Teques de la Universidad Simón Rodríguez. Profesor de Ciencias Naturales, con la mención de Química, egresado del Pedagógico de Caracas. Profesor del área de ciencias en Educación Media, en la Unidad Educativa Magdalena Sofía Barat Caracas. Correo Electrónico: gilberap@gmail.com
} 


\section{INTRODUCCIÓN}

La estrategia convencional de enseñanza o el "dar clases", como se le ha conocido desde siempre, ha sido utilizada por toda la tradición histórica de la educación; representa una manera de transferir información y una práctica oral sostenida en los saberes del docente acerca de un tema en particular. Giroux (1999) la define como un modelo de "transmisión ciudadana" situada más allá de las realidades y relaciones humanas, incluyéndola dentro del paradigma de la "racionalidad técnica" (p. 225).

Esto supone pensar en posibilidades alternas donde la participación del docente se lleve a cabo mediante principios que desarticulen la idea tradicional del acto pedagógico en las aulas de clases. En este sentido, se propone el debate como estrategia de estimulación de la discusión, la cooperación y el conflicto socio - cognitivo; todo ello, con el interés de mejorar la calidad de la práctica, motivar y generar nuevas situaciones de enseñanza que faciliten los procesos de adquisición, elaboración, transferencia y sistematización del aprendizaje.

La utilización del debate cooperativo como estrategia para la estimulación del aprendizaje y el crecimiento personal, conducen a los grupos a la construcción de nuevos saberes, debido a que los estudiantes se exigen así mismo a preguntar y a explicar efectivamente acerca de los temas, siendo el cuestionamiento los orientadores de la efectividad de los conflictos surgidos durante las interacciones. Esta estrategia posee como característica la discusión y la creación de la incertidumbre cognitiva (Johnson y otros, 1999), porque a partir de la controversia o de los puntos de vistas opuestos se origina el conflicto que provoca la reconceptualización de la información en el marco de la ayuda social.

En este orden de ideas, se propone la comparación de dos estrategias de enseñanza aplicadas a grupos diferentes: el debate cooperativo y la exposición basada, exclusivamente, en la intervención verbal del docente.

\section{DESCRIPCIÓN DE LA SITUACIÓN PROBLEMÁTICA}

La educación no ha cambiado lo suficiente como para decirse que se está en un momento de transformaciones profundas. Al contrario, los asuntos relacionados con estos procesos mantienen esquemas y condiciones similares a las del pasado, la escuela ha 
quedado apabullada, oscurecida y descalificada (Bixio, 2008), frente al funcionamiento de otras instituciones y procesos, por lo que el trabajo en las aulas se ha convertido en un gran desafío.

El sistema educativo padece una serie de crisis manifestada en la deserción escolar, la baja o poca preparación docente, la descontextualización del currículo y el abandono cognitivo al cual han sido sometido cientos de niños y jóvenes en escuelas públicas y privadas. Una consecuencia de ello es que los estudiantes que desertan de las escuelas a nivel Educación Básica en su Tercera Etapa, encuentran oportunidad en instituciones orientadas a la Educación de Adultos (Rivero, 2010). Allí revelan serios problemas, no sólo de conducta, sino en el manejo de las actividades cognitivas que les dificulta avanzar, tanto en grado como en conocimientos.

Se descubren jóvenes, que aún con edades avanzadas, presentan dificultades para leer, escribir, analizar textos, hacer síntesis, socializarse con respeto y cordialidad y resolver problemas de índole personal y grupal. La comparación de estrategias para intervenir en el aula, busca entre otras cosas, ensayar modelos que favorezcan la investigación de alternativas pedagógicas más flexibles en cuanto a los medios y mecanismos que permitan al estudiante aprender y les ayude a elevar los niveles cognitivos y de socialización. Por otra parte, la práctica de nuevas metodologías ayuda a la construcción de puentes cognitivos (Flores Ochoa, 2004) que permiten el establecimiento de relaciones y conexiones entre lo que se conoce y lo potencialmente conocible. Un aspecto importante es crear una estructura de convivencia y respeto a las condiciones humanas adversas, reconociéndose las dificultades y limitaciones y valorándose toda revelación y demostración de capacidades.

Tomando en cuenta lo planteado, se formulan las siguientes interrogantes: ¿Cuál es el efecto, en las calificaciones obtenidas en las pruebas, de las estrategias denominadas debate cooperativo y exposición verbal del docente? y ¿Cuál es el alcance, en las aulas de clases, de la aplicación de las estrategias de intervención denominadas debate cooperativo y exposición verbal del docente?

\section{Objetivos de investigación}

Considerando lo anterior se plantean los siguientes objetivos de investigación: 
a. Comparar el alcance, en las aulas de clases, de las estrategias de intervención denominadas debate cooperativo y exposición verbal del docente.

b. Establecer los efectos de la aplicación de las estrategias denominadas debate cooperativo y exposición verbal del docente, en las calificaciones obtenidas por estudiantes del noveno grado de Educación Básica.

\section{ARGUMENTACIONES TEÓRICAS}

\section{La Cooperación y el Aprendizaje}

Cooperar significa compartir experiencias de cualquier naturaleza, supone el trabajo en conjunto y el logro de metas colectivas, es "alcanzar beneficios mutuos a través de una interdependencia positiva entre y con el esfuerzo de todos" (Ferreiro y Espino, 2009 p. 26). Para Adorno (2001) "el trabajo en equipo comporta automáticamente y de forma expresa controles recíprocos" (p. 93), por lo que las relaciones internas, las normas y las características de los grupos definen el alcance de los objetivos, así como la calidad en el manejo de los materiales elaborados para tal fin.

Diversas son las corrientes que han influenciado el aprendizaje y la cooperación, siendo las más destacadas la Escuela Nueva de Freinet (1983), la psicogenésis de Piaget (1981), la interdependencia social de Johnson y Johnson (1999) y (2004), la propuesta socio cultural de Vigotsky (1983), el constructivismo de Coll, (1993), y las teorías de aprendizaje en equipo de Senge (1995), todas ellas han ofrecido aportes que ayudan a comprender este sistema pedagógico de interacción y aprendizaje. La propuesta de trabajar en equipo está basada en las miradas conjuntas que puedan darle los miembros de un grupo a un tema específico, proceso que permite el desarrollo de las capacidades cognitivas y facilita el alcance de los resultados deseados, a su vez, se aprende en la práctica de la discusión y reflexión de los asuntos estudiados.

Johnson y otros (ob. cit) señalan que el aprendizaje cooperativo es el "empleo didáctico de grupos reducidos en los que los alumnos trabajan juntos para maximizar su propio aprendizaje y el de los demás" (p. 14). De igual manera, Slavin (1999) señala que es un conjunto de métodos que tienen como principio básico hacer que los estudiantes aprendan y se hagan responsables del proceso. De manera, que es una propuesta metodológica de intervención donde los estudiantes se dan la oportunidad de informarse y 
aprender con sus pares. Es el compartir experiencias y conocimientos entre iguales, a fin de consolidar el proceso de adquisición y transferencia. Es una herramienta para actuar de manera creativa dentro del aula de clases, a su vez es una alternativa de desarrollo profesional del docente.

El alcance del aprendizaje cooperativo se fundamenta en la convicción de que las interacciones incrementan el dominio de los conceptos fundamentales, ideas u operaciones, y genera la necesidad de la confrontación de los distintos puntos de vistas acerca de un material o tarea específica. King (1990) define este proceso como descentración cognitiva, porque las estructuras intelectuales existentes se reestructuran debido a la confrontación de las ideas, originando nuevas formas y niveles de comprensión. Un buen trabajo en equipo requiere de una planificación que conduzca al estudiante a socializar sus necesidades en lo que respecta el contenido a aprender. El objeto es que se ayuden entre sí para mejorar los resultados en cuanto a la comprensión de los contenidos. A diferencias de las estrategias exclusivas de transmisión de información, el trabajo en equipo permite que los alumnos se manifiesten libremente y desarrollen un autoconcepto de sí mismo (Denegri y otros, 2007).

El producto de las interacciones está relacionado con los grados de ayuda que mutuamente los estudiantes reciben y se otorgan entre sí, a su vez, representa dos situaciones importantes: la primera, el esfuerzo realizado por cada persona, y la segunda, asumir que cada uno de ellos es una parte del conjunto. Esto indica una responsabilidad por parte de cada miembro del equipo a entender la trascendencia del trabajo colectivo. El aprendizaje cooperativo facilita la construcción colectiva de los conocimientos y la corrección mutua de ellos. Esta metodología hace que el aprendizaje se apoye en los compañeros, beneficiándose la construcción de los conceptos y la comprensión de los asuntos estudiados; a su vez se consolida una actitud crítica y analítica, favorecedora en la toma de decisiones con sentido de responsabilidad.

\section{El Debate Cooperativo}

El debate es una práctica que se da en los sistemas democráticos. Su importancia radica, no sólo en la discusión y puesta en común de ideas contradictorias, sino en la posibilidad de permitir espacios sociales compartidos (Bixio, ob. cit), donde la participación y el encuentro deje huellas significativas que permitan la comprensión de lo 
estudiado. Su práctica beneficia la confrontación de las ideas, y ayuda a que los estudiantes mejoren sus niveles de análisis. El debate contribuye a la organización y sistematización de la información, así como el desenvolvimiento en lo que respecta la socialización.

Por lo general, se asocia con discusión, disputas o controversia, pero nunca se relaciona con ejercicios de integración y aprendizaje. Es una estrategia de enseñanza que facilita la confrontación y los conflictos, incluye la clasificación y organización de la información a objeto de aportar conclusiones válidas para comprender los contenidos trabajados. Consiste en exposiciones, defensas y construcciones de posturas y de justificación racional. Durante su aplicación los participantes se sensibilizan ante las ideas opuestas de sus pares, colocándolos en una situación de conflicto conceptual y de incertidumbre: "cuanto más confrontada se siente una persona, mayores son el conflicto conceptual y la incertidumbre que experimenta" (Johnson y Johnson, 2004, p. 147).

Los debates son herramientas estratégicas que facilitan la "curiosidad epistémica" (Jonhson y Jonhson, ob.cit. p.151) que consiste en la necesidad de elaborar constructos teóricos de mayor nivel en la medida en que se lleva a cabo la discusión, conduciendo al estudiante a niveles cognitivos superiores. Además, con estas prácticas en el aula se conjugan una serie de actividades sociales, como la conversación, la discusión, el acercamiento interpersonal y el desarrollo de actividades de comprensión tales como la argumentación, la explicación y la interpretación. Por otra parte, Nérici (1984) señala que el debate es una estrategia exclusiva de "competición intelectual" (p. 298), por lo que su aplicación en el aula de clases, colocan al estudiante en alerta ante la formulación de interrogantes y la construcción de respuestas.

Perkins (1999) señala que es un ejercicio donde la inteligencia es socialmente repartida, ya que la idea es que la controversia, fortalezca la inteligencia y la capacidad comprensiva de los que participan en la discusión.

Debatir cooperativamente es generar situaciones en el aula que conduzcan a que los estudiantes aprendan a discutir y a defender sus ideas, así como a manejar sus emociones para con aquellos equipos que sienten como adversarios. Mediante estas interacciones construyen preguntas y respuestas y socializan con lo que saben y han investigado, todo esto con el objeto de mejorar la calidad del manejo de la información. 
Mediante su ejecución, el estudiante precisa y reconoce las limitaciones en cuanto a la expresión oral, de igual modo experimenta el riesgo de la participación, se defiende y expone sus puntos de vista, sin el temor a "quedar mal frente a sus compañeros", controla las emociones y conductas agresivas, desarrolla habilidades sociales, se incluye en los equipos, se hace habilidoso en la resolución de problemas, en la planificación de actividades y en la toma de decisiones, estos ejercicios de discusión de competencias verbales hacen al alumno creativo y autónomo. En este sentido, Unzué (2012), señala lo siguiente:

En el caso del debate, el alumno no sólo pone en juego la competencia comunicativa lingüística, sino que moviliza también otros sistemas de comunicación para verbal. A esto se añade la imprescindible implicación de la competencia relativa al tratamiento de la información, de la competencia social y ciudadana, de la capacidad de aprender a aprender y del desarrollo de la autonomía e iniciativas personales (p. 14).

Entre las ventajas cognoscitivas, producto de las interacciones en equipos, está el desarrollo mental y social, debido a la existencia del valor de la cooperación entre las personas que intervienen, oponiéndose a las condiciones de aceptación pasiva, conformismo e imitación entre los que interactúan, justificándose el carácter social del conflictos socio - cognitivo que depende del comportamiento de los actores durante las interacciones.

\section{Los Conflictos Socio - Cognitivos}

Con la práctica del debate se dan los conflictos que conducen al progreso cognitivo del estudiante, y favorecen la construcción del conocimiento de los asuntos sociales. Por lo general, todo conflicto facilita los progresos cognitivos (Medrano Samaniego, 1995), siempre y cuando existan condiciones internas iníciales que favorezcan las elaboraciones cognitivas con la ayuda y colaboración de los pares, siendo la socialización el vínculo entre lo que la persona construye internamente acerca del objeto de estudio y lo que este mismo objeto es en realidad.

La aceptación de los errores y las posteriores correcciones ayudan a descubrir y resolver las diferencias en la información, generando procesos, tanto internos como externos, de reconciliación debido a los conflictos ocurridos por la diversidad en los puntos 
de vista. Todo conflicto socio - cognitivo conduce a la reestructuración del conocimiento, porque las contradicciones estimulan el pensamiento. Esto surge a partir de preguntas determinadas que influyen en los procesos cognoscitivos de aquellos que responden, motivando la mejora de la calidad de las respuestas que se dan a las preguntas formuladas y a la acumulación de la información por un largo lapso de tiempo. Las contradicciones generan la construcción de representaciones de un tema, la comprensión y promoción del pensamiento crítico, y permite que las personas exploren y reconozcan el potencial que tienen para hacer inferencias a partir del material estudiado.

Piaget (2009) estableció que las diferencias en las percepciones generan conflictos socio - cognitivos que dan lugar a la construcción social del conocimiento, influyendo, de manera determinante, en los progresos individuales, y este avance se evidencia a nivel colectivo; es por ello, que a medida que los grupos sostengan discusiones profunda acerca de los temas planteados, en esa misma medida cada uno de los miembros participantes mejorarán sus condiciones cognitivas y a su vez el rendimiento académico. Para el autor, tanto la cooperación como el conflicto socio - cognitivo facilitan el desarrollo cognoscitivo y moral de la persona. Por ello, los procesos de aula deben estar fundamentados en ambientes y medios que conduzcan al alumno a la curiosidad epistémica, entendida ésta como el sistema interactivo que facilita la construcción del conocimiento, a partir de todo aquello que representa lo significativo, y donde dicha construcción es alcanzado en el marco de una "naturaleza estrictamente intraindividual" (Carugati y Mugny, 1988 p. 82).

Perret Clermont (1984) considera que las discusiones dadas entre iguales o pares acerca de situaciones específicas generan conflictos de tipo socio - cognitivos, que brindan la oportunidad de mejorar los niveles cognoscitivos del estudiante, por lo que "una de las variables más importantes para que se produzca el progreso, es la posibilidad de intercambiar y confrontar puntos de vista propios con los ajenos (Medrano Samaniego, 1995, p. 178).

En este sentido, Mugny y Doise (1978) señalan que estos conflictos mueven a la persona de un estado cognoscitivo de equilibrio a otro más avanzado, por el choque de perspectivas individuales producto de las discusiones y contradicciones (Rodríguez Arocho, 1999). La persona al relacionar los conocimientos previos con aquellos recién elaborados, hace conexiones internas que causan el aprendizaje, facilitando la organización del material 
de manera significativa, y por otra parte, hace conexiones externas que integran la nueva información en la estructura del pensamiento que posee.

El conflicto socio - cognitivo, así como los diversos orígenes del mismo, sean estos simbólico, emocional, conceptual o hipotético, integra la dimensión social del conflicto a los conocimientos que entran en contradicción para generar actividades socio - cognitivas. Los conflictos socio - cognitivos se basan en la polarización colectiva, o sea en la discusión y los reacomodos cognitivos que se logran a partir de las contradicciones, del afianzamiento y confianza que se adquiere con el reconocimiento de los asuntos aprehendidos, de manera que "los grupos que afrontan abiertamente el conflicto que resulta de sus divergencias interindividuales tienden a producir soluciones cualitativamente superiores, proponen soluciones nuevas y van más allá del nivel del miembro más capacitado del grupo" (Carugati y Mugny, ob.cit p. 81).

\section{La Exposición Verbal del Docente}

Esta estrategia es orientada por la figura del maestro o profesor como eje central del proceso. Al docente se le considera "como la autoridad sobre la materia de estudio, y se ha dado por sentado que los estudiantes aprenden mejor escuchando al maestro y hablando con él" (Stanford y Roark, 1981 p. 100). De manera, que la exposición verbal ha constituido el medio de instrucción centrado en el docente. Es el profesor quien señala a sus alumnos lo que él cree que un estudiante debe saber, ellos se limitan a escuchar y hacer preguntas. Los procesos dados mediante estas prácticas se acompañan de enfoques que intentan o promueven la memorización y repetición de la información. Estos sistemas educativos se sostienen en el orden y la estabilidad ya que los procesos se rigen por teorías educativas que buscan la estructuración y el control (Stenhouse, 1991).

Muchos son los argumentos que se tienen acerca de cómo la práctica tradicional y convencional en el aula de clases, en cuanto a la didáctica se refiere, se ha convertido en un obstáculo para la enseñanza, la adquisición y procesamiento del aprendizaje. La práctica exclusiva de la exposición y la transmisión ha formado a las sociedades actuales, siendo formas seguras de adquisición y transferencia. El hecho de cambiar implica la desestructuración de sistemas y discursos que han sostenido a la educación por muchos años y que han respondido a las creencias de éxitos y logros. Los avances requieren un 
ejercicio mental de des - aprender una serie de condiciones y formas que han conducido, por mucho tiempo, los sistemas educativos.

Se define la estrategia de exposición verbal el sistema donde el docente participa, exclusivamente, en forma verbal durante todo el ejercicio de la enseñanza. La participación del estudiante es mínima sólo se limita a escuchar las explicaciones y descripciones del educador e interviene cuando no comprende algún asunto o cuando el profesor le solicita alguna respuesta ante las interrogantes formuladas en forma verbal. Se fundamentan en principios direccionales y de control de los procesos u eventos que ocurren en el aula, y de reglas de participación uniformes que establecen que los estudiantes aprenden de la misma manera. En este sentido:

(...) esta racionalidad no sólo es objetivado el conocimiento sino que generalmente es reducido al dominio de decisiones técnicas para fines ya decididos. Los fines están afirmados más que explicados como una realidad social. En nombre de la transmisión de creencias abrigadas y valores, este modelo de educación ciudadana termina apoyando, a través de sus metodologías y contenido, la conducta que es adaptable y condicionada, en vez de la activa y crítica (Giroux, ob. cit p. 227).

En este mismo orden de ideas, Flander (citado por Stenhouse, ob. cit) señala que esta estrategia se caracteriza por la exposición de hechos u opiniones de contenidos o procedimientos, donde el docente busca sólo "expresar sus propias ideas, dando su propias explicaciones, o citar a una autoridad diferente a la del alumno" (p. 199).

Por último, esta estrategia compromete el lenguaje como medio exclusivo de enseñanza, excluyendo "la realidad existencial de los maestros, estudiantes y otros sujetos del mundo de la escolarización y de las fuerzas sociales que constriñen y dan forma a la realidad" (Giroux, ob. cit. p. 228).

\section{ASPECTOS METODOLÓGICOS}

El diseño responde a una Investigación de Campo. Se hizo uso del método cuantitativo para comparar el alcance de las estrategias en el aula. Para ello, se preparó un procedimiento basado en un diseño de dos grupos con sujetos no aleatorizados con post pruebas (Ary, Razavieh y Cheser 1990), y aplicado en el área de las Ciencias Biológicas. 


\section{Descripción de la población y de la muestra de estudio}

La población estudiada pertenece a una Unidad Educativa Privada ubicada en la parroquia de Coche en la ciudad de Caracas, con una población conformada por 78 estudiantes. La muestra fue de 42 alumnos, equivalente al 52,84 \% del total de la población en estudio, de ella $20(47,61 \%)$ son del sexo masculino y 22 (52,38\%) del sexo femenino. Entre las características más resaltantes de la misma se mencionan las siguientes: los grupos estudiados pertenecen al noveno grado de la Educación Básica en la modalidad de Educación de Adultos, siendo categorizados como adultos - jóvenes debido a que sus edades oscilan entre los 15 y 18 años, respectivamente. Todos pertenecieron al sistema escolar regular y desertaron por razones de índole cognitivo, motivacional, económicos, familiar, social y de conducta.

Esta población convive en espacios donde se evidencian situaciones de violencia y agresión. Muchos de ellos se encuentran en riesgos, ya sea por su convivencia cercana a las drogas o por su relación con pandillas. Provienen de escuelas donde la estimulación al desarrollo de las habilidades cognitivas y sociales ha sido nula o muy poca, como consecuencia, sienten temor a enfrentarse a desafíos mentales, a discutir, a participar en actividades donde se revelen sus capacidades, presentando serios problemas para expresar cualquier actividad de comprensión.

\section{Hipótesis}

Para la ejecución de la investigación se formularon dos hipótesis: la alterna y la nula. La Hipótesis Alterna quedó definida de la siguiente manera: Las medias de las calificaciones de las pruebas de los estudiantes que participan en la estrategia denominada debate cooperativo son mayores que las medias de las calificaciones de las pruebas de aquellos estudiantes que participan en la estrategia de exposición verbal del docente, y la Hipótesis Nula quedó señalada de la siguiente manera: No hay relación entre las calificaciones obtenidas por los estudiantes y su participación en las estrategias denominada debate cooperativo y exposición verbal del docente. Las variables a medir fueron las siguientes: Variables Independientes son las estrategias de exposición verbal y debate cooperativo, y la Variable Dependiente son las calificaciones logradas por los estudiantes posterior a la aplicación de las estrategias. 


\section{Procedimiento}

Se seleccionaron dos secciones intactas de clases, denominándolos A y B. Los grupos estuvieron conformados por el mismo número de sujetos (21 estudiantes), el grupo A se denominó experimental y al grupo B se le señaló como grupo control. Al grupo A se le aplicó la estrategia de debate cooperativo y, posteriormente, presentaron la prueba escrita. Por otra parte, al grupo B se le aplicó la estrategia de exposición verbal, y luego presentaron la prueba escrita. Cada aplicación se realizó con los mismos contenidos. Repitiéndose el diseño en tres oportunidades en forma seguida para cada grupo.

Con los datos obtenidos se compararon las medias de los grupos, para luego calcularse la desviación estándar de cada situación, a objeto de observar el grado de homogeneidad o heterogeneidad de los datos, de igual modo se evalúo la $t$ de student para corroborar las hipótesis formuladas y realizar las respectivas interpretaciones, a fin de precisar el alcance de las estrategias en el mejoramiento de las calificaciones.

\section{Cuadro 1.}

Diseño aplicado a los grupos Experimental y Control

\begin{tabular}{llc}
\hline \multicolumn{1}{c}{ Grupo } & \multicolumn{1}{c}{ Variable independiente } & \multicolumn{1}{c}{ Postprueba } \\
\hline \hline Experimental & Se aplicó la estrategia de debate. & Presentaron prueba escrita \\
Control & Se aplicó la estrategia de transmisión. & Presentaron prueba escrita \\
\hline \hline
\end{tabular}

Las pruebas fueron las mismas para cada grupo, sus contenidos están señalados en los programas vigentes para el área de Ciencias Biológicas. Se aplicaron de manera individual y al final de la presentación de cada estrategia. Se construyeron mediante la formulación de 20 items de selección simple, con cuatro alternativas de respuestas para cada interrogante. En ellas se examinaron los contenidos referidos a: teoría celular, transporte celular y fotosíntesis y respiración. Es importante destacar que sólo en la primera prueba del grupo control faltó la presentación de un sujeto de estudio, en las demás aplicaciones participó la totalidad de la muestra antes señalada, siempre bajo la orientación e indicaciones del docente de la cátedra, quien es egresado de un instituto pedagógico reconocido en el área de Ciencias Naturales, además posee titularidad en postgrados en el área de las Ciencias de la Educación. Para el procesamiento estadístico y sistematización de los datos se hizo uso del Software SSPS versión 18. 
Posterior a cada aplicación, el docente recogió las impresiones de los estudiantes, tanto con el grupo experimental como con el de control. Esta actividad se llevó a cabo mediante el uso de entrevistas informales de tipo conversacional (McMillan y Schumacher, 2007) orientado a los grupos por separados, siguiendo la lógica de una conversación entre iguales y no mediante los intercambios formales de preguntas y respuestas. La idea del uso de esta técnica fue la de recoger información acerca de los episodios experimentados por los grupos, el chequeo de los sentimientos y emociones manifiestos durante el ejercicio de las estrategias. La información fue recogida por escrito en una libreta destinada para tal fin.

\section{Descripción de las Estrategias de Intervención}

Estrategia de debate cooperativo: Esta estrategia consistió en la siguiente serie de actividades secuenciales: Se invitó a los estudiantes a llevar a cabo una investigación. Para ello, el docente ofreció una serie de pautas, indicaciones e interrogantes orientadoras del proceso indagatorio. Esta actividad se llevó a cabo en el aula de clases, y además, como una manera de ayudar a fijar la información, se evaluaba ya sea con un mapa conceptual, mapa mental, prueba escrita, interrogatorio y exposiciones breves. Lo importante era chequear los contenidos manejados.

En la sesión siguiente se constituía el debate. Se conformaban equipos de cinco miembros como máximo, que debían construir, al menos, cinco interrogantes. Se le daba, a cada equipo, un tiempo para que elaboraran sus materiales de discusión. Posteriormente, se escoge al azar el equipo que inicia la formulación de las preguntas. Los equipos encargados de preguntar seleccionaban, antes de formular las interrogantes, a los miembros de los equipos contrarios responsables en responder. Ante la formulación, los equipos discutían la mejor respuesta y de este modo preparaban al responsable para responder.

Los equipos se calificaron a partir de la calidad de las preguntas y respuestas otorgándole un punto (1) al equipo que respondiera correctamente y cero punto (0) a aquel que no lo hiciera; de igual modo se calificó la calidad de las interrogantes mediante el mismo procedimiento descrito anteriormente.

Después que un equipo formulara sus cinco preguntas, y realizara las discusiones respectivas, se pasaba a otro equipo para que expusiera sus interrogantes, y así, sucesivamente, hasta que todos los equipos cumplieran con el papel de preguntar. De esta 
manera, cada equipo tenía quince oportunidades para responder y cinco para interrogar. Antes de responder los miembros de los equipos debían discutir hasta lograr una respuesta correcta y acorde con la interrogante formulada.

Cada respuesta debía hacerse en un tiempo no mayor de tres minutos. El equipo encargado de formular las preguntas evaluaba las respuestas con argumentos valorando de forma cualitativa cada una de ellas. En ese momento era necesaria la participación del docente para evaluar la pregunta y la calidad de las respuestas, escuchar los argumentos y apelaciones de los equipos, determinar qué equipo merece la puntuación y cuál no, asentar las puntuaciones, ya sea en la pizarra o en la hoja de evaluación, de manera que todos conocieran el record de calificaciones alcanzadas. Al finalizar el proceso los estudiantes presentaban, individualmente, la prueba escrita.

Estrategia de exposición verbal. Esta estrategia se fundamentó en la mayor participación del docente, el cual desarrolló los contenidos y objetivos en forma verbal, explicando y haciendo uso de la pizarra para abordar los temas y describir los procesos y fenómenos. Interrogó en la medida que explicaba los contenidos, elaboró esquemas, aclaró y precisó conceptos, definiciones y temas complejos para luego conducir a los estudiantes a una prueba escrita.

\section{Resultados}

Los resultados obtenidos se presentan en el siguiente cuadro de análisis:

\section{Cuadro 2.}

Resultados de las post - pruebas aplicadas al grupo experimental y control.

\begin{tabular}{ccccccc}
\hline \hline Grupos & Aplicaciones & Medias & Desviación Típicas & $\begin{array}{c}\mathrm{t} \\
\text { estimados }\end{array}$ & $\begin{array}{c}\mathrm{t} \\
\text { crítico }\end{array}$ & $\mathrm{gl}$ \\
\hline \hline $\begin{array}{c}\text { Grupo } \\
\text { Experimental }\end{array}$ & $1 \mathrm{era}$ & 13,0952 & 3,61808 & 16,523 & 1.72 & 20 \\
& $2 \mathrm{da}$ & 14,0000 & 2,60768 & 24,515 & 1.72 & 20 \\
& $3 \mathrm{era}$ & 16,0000 & 2,89828 & 25,219 & 1.72 & 20 \\
\hline \hline Grupo Control & $1 \mathrm{era}$ & 11,6000 & 3,57477 & 14,449 & 1.73 & 19 \\
& $2 \mathrm{da}$ & 10,8571 & 3,60951 & 13,721 & 1.72 & 20 \\
& $3 \mathrm{era}$ & 10,2857 & 4,14901 & 11,305 & 1.72 & 20 \\
\hline \hline
\end{tabular}

Valor de la prueba 0,05 .

La razones observadas de $t$ estimados para cada aplicación del grupo experimental, resultaron ser mayores que los valores de $t$ críticos o los asociados con ciertas probabilidades, esto supone rechazar la hipótesis de nulidad al nivel de significación de $\alpha=$ 
0,05 , de manera que la probabilidad estimada de que esta hipótesis sea verdadera es menor al 5 por 100. Los resultados son lo suficientemente significativos como para señalar que sí existe relación entre las calificaciones obtenidas por los estudiantes y su participación en las estrategias del debate y la exposición verbal del docente. De manera, que tanto las discusiones en y entre los equipos, así como la intervención reiterada del profesor, afectan las calificaciones obtenidas luego de la aplicación de las estrategias a los grupos de estudios.

Esto permite establecer que la discusión en equipo contribuye a generar procesos de adquisición de mayor provecho debido a que se generan conflictos socio - cognitivos que mejoran las condiciones del aprendizaje. Esto hace suponer que el conflicto es una fuente importante para el progreso cognitivo (Carugati y Mugny, ob. cit), es un medio que moviliza a la persona a procesos de reacomodación y adaptación del pensamiento ante nuevas formas de concebir el mundo externo.

La cooperación y la interacción en el aula requieren de situaciones que reestructuren los modos de pensamiento. Durante el conflicto se busca un equilibrio entre los procesos de asimilación y acomodación cognitiva, para alcanzar la adaptación intelectual que se da cuando un sujeto mantiene un proceso deductivo que facilita la asimilación y la acomodación de las estructuras mentales a los datos aportados por la experiencia. La búsqueda del equilibrio supone un conflicto cognitivo donde la interacción entre el sujeto y el objeto pensado mantienen una relación que permite que "lo pensado" sea reestructurado y modificado en sus particularidades. Mientras más diferenciados sean los procesos de asimilación y acomodación, mayores serán los conflictos internos que puedan darse en las personas. Esto supone que el sistema de equilibrio representa un desarrollo mental, que no es más que un paso de menor adaptación o aprovechamiento a un estado superior.

Estos logros académicos pueden ser comprendidos a partir de los procesos de interacción. Las discusiones revelan diferencias entre los miembros de un grupo, estos enfrentamientos aportan información acerca de situaciones específicas y colocan a los que discuten en posiciones divergentes, con puntos de vistas diferentes que ayudan, a través de la discusión, a la modificación de su pensamiento. Los resultados son lógicos a la luz de la teoría del conflicto socio - cognitivo, y puede pensarse que en la medida que los estudiantes discutan más, habrá mayor posibilidad de generarlos y, debido a los sistemas de 
reacomodos, los estudiantes mejoran sus procesos de adquisición y transferencia, evidenciándose en el mejoramiento de las calificaciones académicas.

Un aspecto a destacar es que el grupo experimental mantuvo un ascenso en las calificaciones, al contrario de lo que puede decirse acerca de las calificaciones del grupo control (Gráfico 1). Las calificaciones obtenidas por los estudiantes del grupo control fueron bajas en comparación con el grupo experimental debido al hecho de que la estrategia de exposición verbal no ayuda a mantener al estudiante por mucho tiempo alerta ante las informaciones dadas por el docente. Las interacciones entre los estudiantes es mínima y los asuntos no son tan reiterados con la frecuencia que lo hicieron los del grupo experimental.

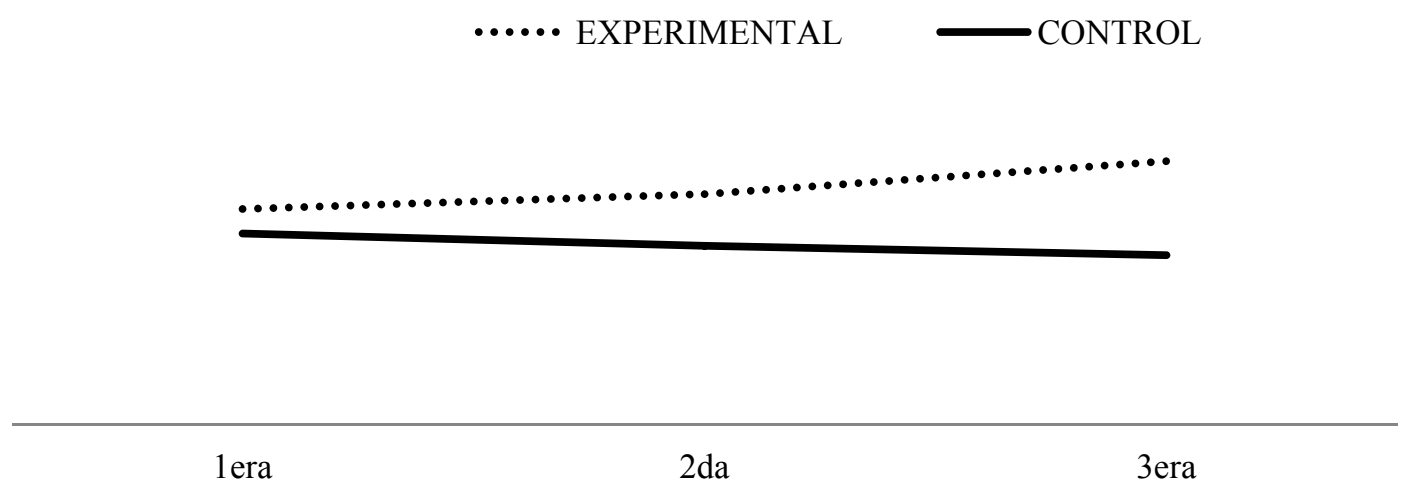

Gráfico1. Comparación gráfica de las calificaciones obtenidas entre el grupo experimental y grupo control, durante los tres momentos de aplicación de las estrategias.

Es de entenderse que el modelo convencional de exposición verbal no ayuda a elevar los niveles cognitivos de los estudiantes, a niveles superiores al que tenían antes de la discusión.

Los estudiantes del grupo experimental estuvieron obligados, permanentemente, a hacerse preguntas de manera reciprocas unos a otros. Se detectó que estas interrogantes provocaron en los otros grupos explicaciones y argumentos con estructuras elaboradas. Los estudiantes que debían responder no se arriesgaban a hacerlo hasta que el grupo, en general, alcanzase un nivel de satisfacción y convencimiento acerca de las respuestas.

Es importante comprender el valor que ofrece la desviación típica, señalando que los datos no presentan mucha variabilidad en relación unos con otros. Por lo general, son uniformes entre ellos, y la información obtenida tiende a ser homogénea. La única que presenta mayor variabilidad es la tercera aplicación del grupo control. Hay una 
homogeneidad en los datos obtenidos en la segunda y tercera aplicación del grupo experimental. Esto conduce a pensar que en la medida que los estudiantes manejen y conozcan la estrategia del debate, su actitud hacia ella es de mayor provecho cognitivo.

El grupo experimental logró un nivel importante de discusión, pero no de manera óptima como, por lo general, se espera de estos procesos. Aún así, presentaron soluciones a los problemas planteados durante los debates. Tanto las discusiones como las respuestas dadas a las interrogantes fueron de calidad, situación reflejada en las post - pruebas. Para muchos problemas dados, las actividades comprensivas no fueron manejas adecuadamente por todos los miembros de los equipos, de manera que esto afectó los resultados de las pruebas.

Por otra parte, la tendencia de las calificaciones en cada una de las evaluaciones logradas en los debates cooperativos es a aumentar. En la medida que los estudiantes mejoran sus condiciones en los debates, en esa misma medida sus calificaciones también tienden a mejorar, de manera que se espera que aquellos equipos que obtengan buenas calificaciones durante el ejercicio del debate, también logren aprobar con calificaciones aceptables las pruebas al final de la estrategia.

\section{Apreciaciones de los estudiantes acerca de las estrategias aplicadas}

\section{Cuadro 3.}

Sistematización de las apreciaciones realizadas por los estudiantes acerca de la aplicación de las estrategias del debate cooperativo y la de exposición verbal del docente.

\begin{tabular}{|c|c|c|}
\hline Estrategias & \begin{tabular}{l}
\multicolumn{3}{l}{ Aspectos que más agradaron a los } \\
estudiantes acerca de las \\
estrategias aplicadas.
\end{tabular} & $\begin{array}{l}\text { Aspectos que menos agradaron a los } \\
\text { estudiantes acerca de las estrategias } \\
\text { aplicadas. }\end{array}$ \\
\hline Debate cooperativo & $\begin{array}{l}\text { - Sintieron que aprendieron mucho } \\
\text { más los temas abordados en } \\
\text { clases. } \\
\text { - Les permitió ayudarse entre sí, } \\
\text { compartir, mejorar las } \\
\text { calificaciones. } \\
\text { - Manejaron temas complejos. } \\
\text { - Los participantes de los equipos } \\
\text { se hacen responsables de sus } \\
\text { actividades. } \\
\text { Todos los miembros del equipo } \\
\text { participan. } \\
\text { Aprobaron, sin dificultad, las } \\
\text { pruebas realizadas después de la }\end{array}$ & 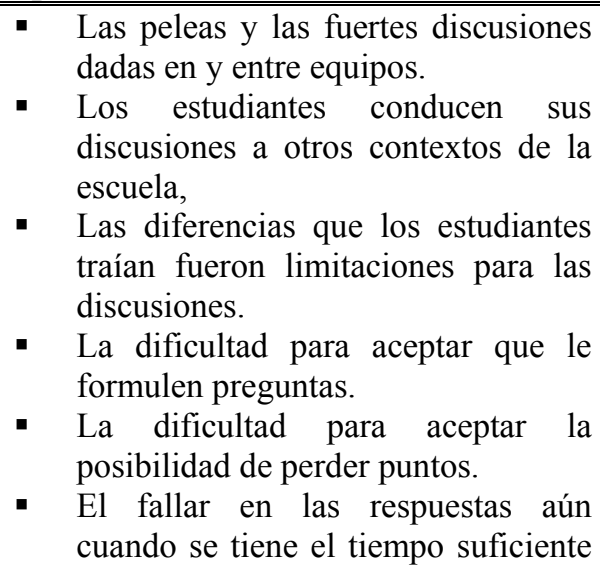 \\
\hline
\end{tabular}




\begin{tabular}{|c|c|c|}
\hline & aplicación de la estrategia. & $\begin{array}{l}\text { para discutir y elaborar, lo mejor } \\
\text { posible, los argumentos y } \\
\text { explicaciones acerca de las } \\
\text { preguntas realizadas. }\end{array}$ \\
\hline Exposición verbal & 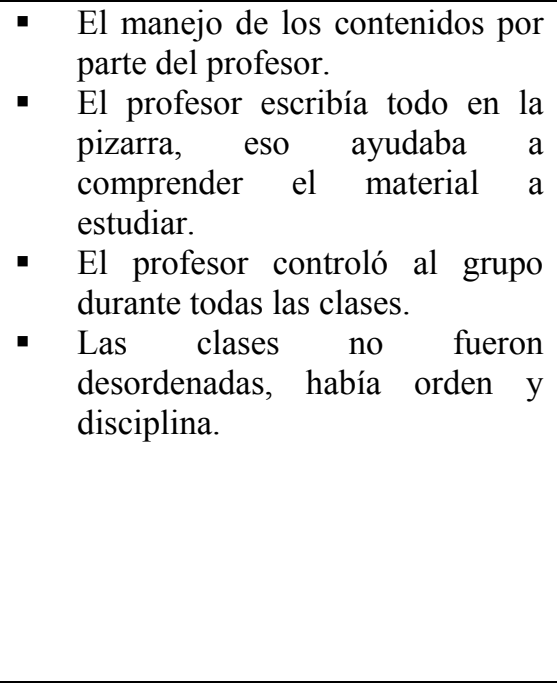 & $\begin{array}{l}\text { - Las explicaciones no se realizaron } \\
\text { de manera pausadas. } \\
\text { - Los tiempos para desarrollar los } \\
\text { temas eran muy cortos. } \\
\text { En ocasiones el docente se aislaba } \\
\text { de los temas, creando confusión. } \\
\text { Algunos contenidos generaban } \\
\text { cansancio. } \\
\text { Las actividades de investigación, las } \\
\text { cuales se hacían posterior a la clase, } \\
\text { no eran evaluadas, pero sus } \\
\text { contenidos sí eran utilizados en las } \\
\text { pruebas. } \\
\text { Los contenidos trabajados se hacían, } \\
\text { a veces, incomprensibles por lo que } \\
\text { no era suficiente una clase para } \\
\text { desarrollarlos. }\end{array}$ \\
\hline
\end{tabular}

\section{CONCLUSIONES Y RECOMENDACIONES}

Las conclusiones que se desprenden de esta investigación, sólo son aplicables a la muestra trabajada. Es de precisar que la misma no es aleatoria, por lo que sus resultados no pueden ser generalizado. En este sentido, se señala lo siguiente:

El debate cooperativo facilita que los estudiantes mejoren sus calificaciones en comparación con la estrategia de exposición verbal del docente. Esto indica que la relación entre pares es efectiva a diferencia de las pocas interacciones que se dan en las clases donde el educador, exclusivamente, lleva a cabo la explicación.

La estrategia del debate, en el marco del aprendizaje cooperativo, contribuye a que los estudiantes elaboren interrogantes, respuestas y argumentos de calidad y en equipos, a su vez ayuda a la disminución de la violencia en el aula aunque genera discusiones y conflictos que colocan el valor de la amistad en riesgo. La estrategia genera este tipo de reacciones en los estudiantes, pero la adecuada mediación del docente facilita la comprensión y el manejo de los conflictos. Facilita la participación de todos los miembros del aula en las discusiones y en las decisiones. Esta actividad implica elaboraciones verbales y escritas, siendo efectivas para desarrollar procesos de interpretación y comprensión de los contenidos. A su vez, se condicionan los conflictos socio - cognitivos y su exteriorización, mejorando los niveles de elaboración de contenidos a partir de la 
construcción colectiva, ya sean de preguntas, respuestas y de argumentos que conducen a la resolución de problemas.

El entrenamiento previo, en cuanto a la elaboración de preguntas y la investigación de los contenidos, contribuye a que los estudiantes elaboren materiales de alto nivel. La estrategia hace eficaz la transmisión de los contenidos, ayuda a controlar los niveles de elaboración de respuesta y su efectividad, de igual manera genera espacios para desarrollar valores tales como: la ayuda mutua, la cooperación y la responsabilidad, siéndose efectiva su aplicación para aquellos estudiantes renuentes a participar en clase. Es importante destacar que su aprovechamiento aumenta en la medida en que su aplicación se hace frecuente y de forma reiterada.

Al intervenir en cualquier equipo, el estudiante mantiene un conocimiento que le ayuda a crear inferencias a partir de una nueva información. El cuestionamiento y la explicación crean niveles entre un conocimiento existente y otro que se hace más significativo y que a la vez es nuevo, de esta manera se construyen redes cognoscitivas que ayudan a la memoria a recuperar textos escritos u orales. En este sentido, los conflictos socio - cognitivos y las contradicciones surgidas en los equipos generan situaciones de aprendizaje significativo desarrollando un pensamiento crítico.

Los resultados conducen a explicar que las interacciones sociales dadas en los equipos que construyen preguntas y respuestas, contribuyen a la construcción del conocimiento. Las diferentes percepciones generan conflictos, a su vez las debidas reconciliaciones se demuestran al momento en que el estudiante explica, con sus herramientas verbales y cognitivas, el modo en que elaboró sus constructos.

El trabajo en equipo crea las condiciones para que los estudiantes aborden y se relacionen tanto con los problemas, como con el resto del equipo. Genera aprendizajes, poniendo a prueba la comprensión de los materiales y el reconocimiento de las dimensiones de las situaciones problemáticas, además propicia un ambiente de igualdad $\mathrm{y}$ responsabilidad compartida. Por otra parte, permite que los estudiantes se cuestionen y generen mayores y mejores explicaciones de los temas y problemas, elaboren hipótesis y respuestas claras a temas concreto, de la misma forma ayuda a justificar los hechos y los contenidos de los materiales, reforzándose el "por qué" de los mismos a partir de las 
relaciones con el conocimiento anterior, facilitando construcciones con sentido explicativo y analítico que mejora el logro de los aprendizajes.

El debate y el cooperativismo hacen que los estudiantes experimenten procesos creativos. Facilita la libertad en las construcciones colectiva; los estudiantes pueden controlar los contextos donde se desenvuelven, aumentan su motivación intrínseca y refuerzan el sentido de libre determinación; esta estrategia promueve la producción espontánea a partir de una mejor comprensión de los materiales, siempre orientada hacia dos situaciones específicas: (a) la elaboración, en un alto nivel de interrogantes, respuestas y teorías y (b) la búsqueda de nuevas formas de pensar sobre los materiales con que se trabajan en aula.

Durante el desarrollo de la estrategia de exposición verbal del docente no se evidenciaron situaciones conflictivas entre los estudiantes, ya que las interacciones entre ellos fue muy poca, el docente tuvo la mayor participación en cada jornada. Por lo general, esta estrategia se sostiene en las siguientes condiciones: (a) la función del docente es la de "dador de clases", su participación en el aula es mayor que la de los estudiantes; (b) el docente tiene la mayor responsabilidad, sobre él reposa la planificación y dirección de la enseñanza, por lo que el proceso es predecible; (c) las decisiones acerca de los procesos dados en el aula, así como la orientación de todo el sistema está controlado por el docente. El docente no negocia, más bien impone su criterio y (d) las discusiones son pocas, muchas veces no ocurren. El único mecanismo que tiene el estudiante en el aula, para adquirir la información, es a través de la atención puesta en lo que el docente explica.

En la medida que se aplican estrategias opuestas a la estrategia de exposición verbal, los estudiantes identifican las diferencias y son capaces de mejorar las condiciones de aprovechamiento. Esto se evidenció durante la aplicación de las dos estrategias diseñadas. En la estrategia del debate cooperativo los estudiantes descubrieron un mecanismo, no sólo para aprender, sino para mejorar las calificaciones en relación con sus compañeros y a su vez precisan las diferencias con otras estrategias, valorándolas ambas según sus criterios y experiencias. Ocurre lo contrario con los estudiantes que participaron en la estrategia de exposición verbal, sin conocer otras posibilidades se le hace difícil comparar, perciben el "dar clases" como la estrategia donde el docente debe demostrar sus habilidades y destrezas en el campo. Una diferencia significativa en esto es que en el debate cooperativo, son los 
estudiantes los que deben demostrar sus capacidades, tanto de adquisición como de transferencia y sistematización, y en la estrategia de exposición verbal las capacidades deben ser demostradas por el docente, los estudiantes sólo demuestran su capacidad de adquisición.

Se recomienda, en el marco de la aplicación del debate cooperativo, que se establezcan, desde el inicio, una serie de normas que conduzcan la discusión y el sistema de evaluación. Se evite durante el desarrollo de la estrategia el desencadenamiento de situaciones de violencia, siendo necesario el ejercicio de la autoridad con disciplina y respeto en función de que las normas se cumplan a cabalidad y se revisen los materiales escritos a partir de los significados surgidos de las interacciones, así como el papel del docente ya que esto implica el cambio de "dador de clase" a orientador, facilitador y supervisor del proceso.

\section{REFERENCIAS}

Adorno, T. W. (2001). Epistemología y Ciencias Sociales. Madrid: Cátedra.

Ary, D., Cheser J. L., y Asghar, R. (1990). Introducción a la Investigación Pedagógica (2da edic.). México: McGraw - Hill.

Bixio, C. (2008). ¿Chicos aburridos? El problema de la motivación en la escuela. México: Limusa.

Carugati, F. y Mugny, G. (1988). La teoría del conflicto sociocognitivo. En: Mugny G. y Pérez J. (Dirs). Psicología social del desarrollo cognitivo. Anthropos: Barcelona.

Coll, C. S. (1993). Constructivismo e Intervención educativa ¿Cómo enseñar lo que se ha de construir? En: Beltrán, J. A., Bermejo, V., Prieto Ma. D. y Vence, V. (Dirs). Intervención Psicopedagógica (pp. 230 - 247). Madrid: Pirámide.

Denegri, M. C., Opazo, P. C. y Martínez, T. G. (2007). Aprendizaje cooperativo y desarrollo del autoconcepto en estudiantes chilenos. Revista de Pedagogía 28 (81) 13 41.

Ferreiro, G. R. y Espino C. M. (2009). El ABC del aprendizaje cooperativo: Trabajo en equipo para enseñar y aprender. (2da. edic.) México: Trillas.

Flores Ochoa, R. (2004). Evaluación pedagógica y cognición. Bogota D.C.: McGraw - Hill Interamericana, S. A.

Freinet, E. (1983). Nacimiento de una pedagogía popular. Barcelona, Laia. 
Giroux, H. (1999). Teoría y resistencia en educación. Una pedagogía para la oposición. México, D. F.: Siglo veintiuno editores.

Johnson, D. y Johnson, R. (1999). El Aprendizaje juntos y solos. Buenos Aires: Aique Grupo Editor S.A.

Johnson, D. y Johnson, R. (2004). Cómo reducir la violencia en las escuelas. Buenos Aires: Paidós.

Johnson, D., Johnson, R. y Holubec. (1999). El Aprendizaje cooperativo en el aula. Buenos Aires: Paidós.

King, A. (1990). Enhancing Peer interaction and learning in the classroom through reciprocal questioning. American Educational Research Journal. 27 (4) 664-687.

McMillan, J. H. y Schumacher, S. (2007). Investigación cualitativa (5ta. edic.) Madrid: Perarson Educación, S. A.

Medrano Samaniego, C. (1995). La interacción entre compañeros: el conflicto sociocognitivo, el aprendizaje cooperativo y la tutoría entre iguales. Revista Interuniversitaria del formación del profesorado (23) 177 - 186.

Mugny, G. y Doise, W. (1978). Socio - cognitive conflict and the structure of individual and collective performances. European Journal of Social Psychology, (8) 181 - 192.

Nérici, I. G. (1984). Hacia una didáctica general dinámica. Buenos Aires: Kapelusz.

Perret Clermont, A. N. (1984). La construcción de la inteligencia en la interacción social. Visor: Madrid.

Piaget, J. (1981). Psicología y Epistemología. (5ta. edic.). Barcelona: Ariel.

Piaget, J. (2009). La psicología de la inteligencia. (Foix, J. C. trad.). Barcelona: Crítica S.L.

Perkins, D. (1999). La escuela inteligente. Barcelona: Gedisa.

Rivero, J. (2010). Aprender a lo largo de la vida en contextos de inequidad. En: Marchesi, A. y Poggi, M. (Dirs.) Presente y futuro de la educación iberoamericana. (pp. 181 202). [Libro en línea]. Madrid: Agencia Española de Cooperación Internacional para el Desarrollo (AECID). Fundación Carolina. Disponible [Consulta: 2013, enero, 15].

Rodríguez Arocho, W. (1999). El legado de Vigotski y de Piaget a la educación. Revista Latinoamericana de Psicología 31 (003) 447 - 489.

Senge, P. (1995). La quinta disciplina. El arte y la práctica de la organización abierta al aprendizaje. Barcelona: Juan Granica S. A. 
Slavin, R. (1999). Aprendizaje Cooperativo. Teoría, Investigación y Práctica. Buenos Aires: Aique Grupo Editor S.A.

Stanford, G. y Roark, A. E. (1981). Interacción humana en la educación. Méxixo: Diana.

Stenhouse, L. (1991). Investigación y desarrollo del curriculum. (3era. edic.) Madrid: Morata.

Unzué, U. A. (2012). Argumentar para debatir. Una propuesta para todas las áreas de secundaria $\left(\mathrm{N}^{0}\right.$ 2) [Libro en línea]. Gobierno de Navarra: Colección Biblioteca Escolares. Serie Naranja. Disponible: http://dpto.educacion.navarra.es/publicaciones/pdf/argumentar.pdf [Consulta: 2012, febrero 03].

Vygostky, L. (1983). Pensamiento y lenguaje. Buenos Aires: La Pleyade. 